\title{
Reasons for Failure in Endoscopic Sinus Surgery
}

\author{
Scott M. Graham
}

Published online: 12 January 2013

(C) Springer Science+Business Media New York 2013

\begin{abstract}
Despite advances in instrumentation, imaging and the understanding of the pathophysiology of some diseases, "failure" of endoscopic sinus surgery continues to be an important problem. The impact of failed surgery, whether economic, emotional or health-related, is considerable. Technical factors continue to play an important role in failed surgery. In others, poor patient selection is a key issue, particularly in the difficult area of facial pain. In other patients, systemic diseases, including immune deficiency, may contribute to a poor result. In other patients still, repeated "functional", "ostio-meatal complex" surgery has little prospect of success, particularly when it has been unsuccessfully employed on prior occasions. In this patient group, more extensive surgery may need to be considered. As a corollary of this argument, some patients with advanced disease-predictably difficult to treatmight benefit from more surgically aggressive treatment, even at initial presentation.
\end{abstract}

Keywords Sinus surgery failure $\cdot$ Complications $\cdot$ Facial pain $\cdot$ Headache $\cdot$ Immune deficiency $\cdot$ Modified osteoplastic frontal sinus surgery

\section{Introduction}

Failed endoscopic sinus surgery represents a distressing investment of a patient's time, emotion, money and discomfort culminating in an unsuccessful outcome. For the individual patient, this is a uniquely personal anguish. For

S. M. Graham $(\bowtie)$

Department of Otolaryngology Head and Neck Surgery,

The University of Iowa, Iowa, IA, USA

e-mail:scott-graham@uiowa.edu the surgeon, quite apart from the personal impact an unsuccessful result might have, there is also a considerable reputational risk inherent in bad results or failure, to say nothing of medico-legal concerns. A bad result from a patient perspective may simply represent the almost inevitable natural history of a disease-polyp recurrence perhaps-in Samters Triad. Whether a surgical outcome in such circumstances is perceived by the patient as "surgical failure" or as a reflection of the difficulties of the underlying disease may depend in large measure on the preoperative counseling provided to the patient and also on exhausting other therapies prior to the decision for surgery. Other surgical failures, such as certain operative complications, will be dramatically and immediately obvious [1].

\section{Technical Factors}

Clearly technical factors contribute to a number of cases of surgical failure. The complexities of frontal sinus surgery demand a chapter or even a book all of their own. Likewise, most technical difficulties involving incomplete ethmoidectomy and extra-mural ethmoid pneumatization have received wide coverage in the literature. Image-guided surgery may be helpful in resolving some of these technical issues. Surgical strategies involving different techniques for maxillary antrostomy and sphenoidotomy have also received a good deal of attention.

Less easy to examine, and much less frequently talked about, are more nebulous concepts influencing the outcome of sinus surgery. Actual surgical technical ability is difficult to measure and likely varies between surgeons. A more nebulous concept still is the "desire to do a good job". In practical terms this often involves devoting an appropriate amount of time to a given patient's operation. Complex 
cases often require meticulous, thorough, time-consuming dissection-surgery that may not easily be achieved within the confines of a busy operating list crammed with multiple patients.

Surgical success is facilitated by optimal exposure and visualization. Visualization depends in part on the availability of appropriate instrumentation and angled telescopes. Septal surgery and turbinate surgery, such as concha bullosa reduction, play important roles in exposure in selected patients. Visualization also depends in large measure on blood loss. Almost everything we do in sinus surgery is aimed at reducing blood loss and by implication enhancing visualization, and therefore the precision of dissection and surgical safety. Appropriate pre-surgical treatment with antibiotics and oral steroids is important in some patient groups, as is the exclusion of a bleeding history or the safe cessation of drugs like aspirin. Operative influences include not only anaesthetic choices [2], head of bed elevation, nasal packing and judicious injection, but also the requirement to allow an appropriate amount of time for vasoconstrictor medication to work.

\section{Scope of the Problem}

There are said to be in excess of 30 million Americans with chronic rhinosinusitis [3]. Whether there are in fact 30 million people with actual appropriately diagnosed chronic rhinosinusitis is open to debate, as the 30 million figure may well include vasomotor rhinitis, allergic rhinitis and a variety of facial pain syndromes. Nonetheless, the dollar expenditure by patients in search of a "sinus cure" is huge-estimated to be in the excess of six billion dollars annually in the 1990s [3]. It is difficult to imagine that the current dollar figure is lower than this. In pursuit of curing the substantial number of patients with 'sinus-related complaints' an estimated 500,000 sinus surgeries are performed annually in the United States [4]. Depending on the rigor with which success or failure is defined, failure rates of $15-50 \%$ have been quoted for sinus surgery in published series. Endoscopic sinus surgery has been widely performed in the United States for the last 20 years or so, and while the precise number of "failed surgeries" is uncertain, the number is likely to be large. Failed surgery and persisting symptoms have a substantial impact on quality of life and we need look no further than the internet for testimony to the variety of 'cures' sold to patients with persisting symptoms.

\section{Facial Pain}

Indications for surgery in patients with chronic facial pain are among the most controversial in all of rhinology. Facial pain is a common complaint, and we also know that incidental sinus CT changes are common in the general population $[5,6]$. Despite considerable evidence to the contrary, chronic facial pain as an isolated complaint is often treated as 'sinusitis' in the wider medical community. Patients often appear in the otolaryngologist's office sure that their sinuses are the cause of their facial pain and headache, and equally certain that surgery or indeed more surgery will be required to fix it. Certainly, there are surgeons willing to oblige this patient population, and there is even a report extolling the virtues of operating on radiologically normal sinuses for facial pain [7]. Add to these general difficulties other issues involved with septalmucosal contact points, conchae bullosa $[5,6]$ or incidentally diagnosed osteomas [8•], and this area is confused indeed. Enthusiasts for the central role of the sinuses and nasal cavity in facial pain will proclaim substance-P-initiated difficulties [9] or intermittent ostio-meatal complex closure, not visualized on imaging, as causes for surgically treatable facial pain. The alternative point of view is that facial pain is a discrete neurological entity unrelated to most long-standing intranasal findings [10, 11]. A rational examination of these distinctly different points of view is crucial for treatment planning. Should the patient have surgery for some small incidental sinus mucosal change, seen in perhaps $40 \%$ of the normal population, or should the patient be referred for initial neurological assessment and management? From a patient perspective, unsuccessful surgery performed for an indication of 'facial pain' is as much a surgical failure as any other unsuccessful operation. Sometimes its tempting to 'give surgery a try,' 'just in case' it might be helpful for an often severe problem. The difficulty with this approach-notwithstanding the risks inherent in any sinus operation-is that surgery, in a poorly understood fashion, may change the quality of the pain to an even more disturbing neuropathic variety in a percentage of patients.

Our own practice is to refer patients with CT-scan- and endoscopy-negative facial pain for specialist neurologic review. Communicating the need for neurological assessment is sometimes a difficult conversation to have with a patient. Many patients are frankly incredulous that their pain might be due to something other than their sinuses. Patient's feelings on this matter are understandable. The concept of "sinus headache" is widely entrenched in popular culture. A visit to the 'sinus aisle' at any grocery store reveals a wealth of proprietary medication mixes aimed at curing the sinus pain graphically depicted on the medication packaging. Furthermore, patients have often made a considerable financial investment in sinus treatment-antibiotics, sprays, allergy therapies, and even thusfar-unsuccessful surgery. Patient resistance to the concept of non-sinus causes of their facial pain and headache is 
therefore understandable. Many patients are so outraged that they have stumbled upon such a poorly informed surgeon that they immediately seek out a compliant otolaryngologist, and certainly never consider for a moment keeping the neurology appointment.

Paulson and Graham [11] reported 104 consecutive patients seen with CT-scan- and endoscopy-negative facial pain who were referred to neurology for treatment. Twenty-nine patients did not attend the neurology appointment. Forty of the remaining 75 patients were seen on multiple occasions. Of the patients seen by the neurologists, $4 \%$ were given a new diagnosis of serious intracranial pathology. Migraine (37\%), analgesic rebound headache $(17 \%)$, chronic daily headache $(17 \%)$, and obstructive sleep apnea were the commonest diagnoses. At a mean follow-up of 10.5 months, $58 \%$ of the patient group had significantly improved on disease-specific pharmacotherapy. $79 \%$ of patients with prior unsuccessful sinus surgery performed for facial pain improved on medical treatment. Patients with a pristine CT scan of their sinuses and no major septal deviation were statistically more likely to respond to medical therapy than patients with septal deviation and minimal mucosal change.

A 2003 publication by Welge-Luessen et al. [12] examined the benefit of endonasal surgery in patients with contact points and a diagnosis of migraine or cluster headache previously made by a neurologist. The 20 patients in the study had headache that was resistant to pharmacological therapy directed by the specialist neurologists. Two years after the surgery, $85 \%$ of patients noted 'improved symptoms'. Interestingly, this number fell to $65 \%$ at 10 years. The authors continue to recommend initial neurological evaluation in these patients but have highlighted intriguing surgical treatment possibilities for a very carefully selected subgroup of headache sufferers.

Extra impetus has been added to this argument by the evolving issue of surgical treatment of patients with a known diagnosis of migraine. Two studies address surgery for patients with a diagnosis of pharmacologically refractive migraine and radiographic sino-nasal mucosal contact points. Behin et al. [13] reported generally favorable results as measured by a reduction in headache frequency, severity and headache related disability in 21 patients over 5 years. Successful application of topical anaesthesia to the contact area prior to operation was required for inclusion in their surgical series.

Guyuran et al. [14•] reported 5-year outcomes of surgical treatment of migraine headaches. In a review of their experience from Cleveland, the authors cited a figure of 30 million Americans who suffer from migraine-curiously a very similar figure to the number of people in the USA said to suffer from chronic rhinosinusitis. A variety of candidate surgical trigger points were identified and initially treated with botulinum toxin A (Botox; Allergan, Irvine, California). Control patients were injected with saline. Eventual surgical treatment for intra-nasal contact patients was septoplasty and turbinectomy. Improvement was noted at 12 and 60 months, with $75 \%$ of patients identified as having trigger sites that included the nose. Only 3 of the 89 patients, however, had treatment for an isolated intranasal trigger point.

\section{Immune Deficiency}

In 2001, Chee et al. [15] demonstrated a surprising incidence of immune dysfunction in patients with refractory sinusitis treated in a single tertiary care institution. Patients with HIV/AIDS, cystic fibrosis, Wegener's granulomatosis, Churg-Strauss, a history of chemo-therapy, allergic fungal rhinosinusitis or primary ciliary dyskinesia were excluded from their study population. Refractory sinusitis was diagnosed in patients with more than three episodes of appropriately diagnosed infection in the year prior to inclusion. The 79 patients in the study had an average of three sinus surgeries prior to diagnosis and were mostly middle-aged women. Their average Lund-McKay CT score was 11.2, and auto-immune disease was present in a quarter of the studied group.

This highly selected patient population demonstrated an incidence of common variable immunodeficiency of $9.9 \%$-strikingly higher than the often quoted likelihood of CVID in the general population of 1:50,000-1:100,000. Selective IgA deficiency was found in $6.2 \%$ in this patient group, compared with $0.3 \%$ of the general population. More recently, Al Qudah et al. [16•] reported on a similar patient cohort studied between 2001 and 2009. Since the Chee et al. [15] study, a new 'immune deficiency diagnosis' had become widely accepted, that of antibody deficiency with normal immunoglobulins. This condition is also known as "functional antibody deficiency" or "specific antibody deficiency." This diagnosis rests on demonstration of a lack of appropriate antibody response on paired serologic testing to antigenic challenge in 50 or $70 \%$ (in children or adults respectively) of the serotypes contained in an unconjugated polysaccharide vaccine (Pneumovax, Merck Sharp and Dohme, West Point, PA). Of the 51 patients studied, 34 (or $67 \%$ ) were diagnosed with specific antibody deficiency. While both of these studies suffer from the limitations inherent in retrospective analyses, inasmuch as the immune work-up was patientbased and non-uniform, they do serve to identify the high incidence of immune dysfunction in the refractory sinusitis population.

In identification of patients at risk who would benefit from immune testing, the acronym CURSE is helpful. That 
is, patients with chronic, unusual, recurrent or severe infections should be evaluated. Quantitative levels of serum IgG, IgA, IgE should be obtained, as these may reveal a diagnosis of CVID. If these levels are normal and clinical suspicion remains, functional antibody responses to an unconjugated polysaccharide vaccine should be obtained. Making the diagnosis of immune deficiency is useful from a number of view points. Patients often appreciate a firm diagnosis, particularly after a lengthy and complicated period of ill-health. Secondly a diagnosis of immune deficiency often tempers enthusiasm for further surgery or at least imparts realistic expectations for further operative intervention. Thirdly useful treatments exist for patients with a diagnosis of immune dysfunction and continuing sinus symptoms.

Treatment in such patients is often undertaken using a tiered approach. Initial treatment includes local therapy incorporating saline irrigations, topical steroid sprays and surgery when needed. Second tier therapy comprises antibiotic prophylaxis. Lastly, immune replacement can be provided by IVIG or more recently subcutaneous immunoglobulin.

\section{Need for More Extensive Surgery?}

For the vast majority of patients, 'functional' endoscopic surgery provides their best chance for improvement with the least likelihood of an adverse outcome. The minimal surgery required to fulfill the goals of treatment is planned. Uninvolved tissue is spared, mucosa is preserved, and muco-ciliary clearance is restored or maintained. More extensive, non-physiologic but historically important surgery, such as modifications of the Caldwell-Luc procedure, naso-antral windows, extensive turbinate removal, or external approaches, are seldom performed. These older procedures may involve skin incisions, are often destructive of tissue, and have predictable and unwelcome sequelae. An added concern with extensive removal of intra-nasal tissue is the specter of the poorly understood socalled "empty nose syndrome" [17]. There is, however, a small group of patients for whom ostio-meatal complex surgery has not proved to be all things for all symptoms, and in whom repeated 'functional' procedures have limited prospects of success [17]. In other patients, conventional endoscopic techniques and instrumentation do not provide access to all areas of the diseased sinus. As a result of these concerns, there has been a re-examination of the selective application of elements of more traditional surgery in small patient populations.

One area of particular interest has been the maxillary sinus. Alby et al. [18••], from Romania, reported on 96 patients with nasal polyps and total maxillary sinus CT opacity who were randomized to receive conventional middle meatal antrostomy or middle meatal as well as inferior meatal antrostomies. The patients receiving the combined surgical procedure had better results 1 year after surgery as measured by a variety of parameters.

Extension of the maxillary antrostomy to the floor of the nose with resection of the posterior half of the inferior turbinate, the so-called "endoscopic maxillary megaantrostomy", was described by Cho and Hwang [19]. Twenty-seven patients who had failed prior procedures had 42 mega-antrostomies performed over a 27 month period. $59 \%$ of the group described improvement at a mean follow-up of 11 months. The goal of retention of the anterior portion of the inferior turbinate is to reduce the likelihood of post-operative nasal dryness. No cases of naso-lacrimal system injury were described. As might be expected, the symptom which responded most poorly to this procedure was cheek pain after a prior Caldwell-Luc surgery.

A continuing difficulty in endoscopic sinus surgery is the common clinical situation of extensive polyps, diffusely distributed through the maxillary sinus. Custom irrigation devices are helpful in removing fungal debris from the maxillary sinus; however, polyps in difficult-toaccess areas of the sinus are challenging to surgically remove, even when operating through a wide maxillary antrostomy with an angled microdebrider blade and angled telescope. Surgical modifications involving extra dissection have been described to allow for more extensive disease removal in the hope of reducing the likelihood of failed surgery. A "mowing" operation, in which extra clearance of sinus polypoid change is achieved by introduction of a microdebrider blade through the canine fossa, was described by Sathanathar et al. [20]. Injury to branches of the anterior superior alveolar nerve and other complications of the canine fossa opening are the conceptual drawbacks of this procedure. Performing the canine fossa puncture at the intersection point of imaginary lines drawn from the midpupillary point and floor of the pyriform aperture was said by Robinson and Wormald [21] to minimize complications. Inevitably, complications still occur, and while ultimately resolving, as reported by Singhal et al. [22], these complications can cause clear difficulties in the short term. Generally good results using canine fossa puncture and additional dissection were reported by Serberling et al. [23] in a series of 97 patients with severe maxillary disease and a mean follow-up of 27.8 months.

Challenging these results, Lee et al. [24••], from Korea, reported no benefit from the procedure. Furthermore, complications of canine fossa puncture were reported in $54 \%$. In an effort to quantify the potential benefit of added canine fossa dissection, Feldt et al. [25] used a model incorporating minced cat food to represent sinus debris. A combined procedure incorporating a canine fossa approach 
yielded a further $1 \mathrm{cc}$ of cat food compared with isolated trans-nasal dissection. Our own practice is to introduce the microdebrider blade through the inferior meatus when extra dissection is called for. The canine fossa puncture provides a superior "straight line" approach, however we feel that the inferior meatal approach provides a better balance between further dissection and possible adverse sequelae [17].

More radical surgery still is advocated by a group of surgeons from the Netherlands in very select circumstances. Wreesman et al. [26] reported on 82 patients over the 10-year period to 1997 who received their version of the Denker's procedure as "last resort" surgery. These patients had such diagnoses as Samter's Triad or cystic fibrosis, with no reasonable prospect of re-establishment of normal muco-ciliary clearance. After such surgery, $84 \%$ of patients reported improved symptoms. No cases of "empty nose" syndrome were reported. The Dutch investigators recommend this procedure for certain carefully selected patients who have failed more 'functional' surgery.

Videlaar et al. [27] studied a similar group of patients in a prospective fashion. The surgical goals were to enhance drainage and aeration and to provide access for cleaning or topical therapy. With an average of six prior surgeries, this patient group was again beyond the goals of more conventional therapy. This more radical surgery comprised $3 \%$ of the sinus surgery performed in the authors' institution between 1999 and 2002. Generally good results were noted at 1- and 2-year follow-up.

Modified osteoplastic frontal sinus surgery continues to be important for a small number of patients. Alqudah and Graham [28•] recently reported their experience involving 17 patients with diverse pathologies, including laterally located mucoceles, osteomas, osteomyelitis and sino-cutaneous fistula. Unequivocally, endoscopic procedures are the first choice for the overwhelming majority of patients with frontal sinus pathology. The popularity of Draf III procedures has further reduced the need for "external frontal sinus surgery". The authors report a modification of the traditional osteoplastic technique with full elevation of the pericranium, maintaining its potential for use as a flap should this be required. No complications were reported with this surgical modification.

An area receiving attention, particularly in Australia, is the question of whether more aggressive surgical treatment of, in particular, recurrent polyps in the frontal recess reduces the need for further revision surgery. Bassiouni and Wormald [29] recently reviewed patterns of polyp recurrence in 299 patients with CRS and NP from 2003 to 2010. In particular, they were interested in examining the role of the extent of frontal sinus surgery (Draf 2a vs. Draf 3) on polyp recurrence rates. At 6 months, polyp recurrence was noted in $35 \%$ of patients who had undergone Draf 3 procedures and in $42 \%$ of patients who had received more 'standard surgery'. Revision rates, which of course incorporate a subjective element in surgical decision-making, were significantly lower in the Draf 3 groups.

One hypothesis that addresses this apparent improvement in patients undergoing more extensive surgery is the concept of the, "inflammatory load". More extensive surgery reduces the inflammatory mass of eosinophils, while also providing ventilation for the sinus, and also probably providing better access for topical therapies. Eosinophils are thought to be most densely located at the base of nasal polyps, and more extensive polyp removal down to basement membrane provides maximal removal of inflamed tissue-certainly more than only sinus ostial enlargement [30॰].

If more extensive surgery is helpful in cases of polyp recurrence [29], an important question is whether the judicious initial use of extensive surgery might be of benefit in selected patients. One might hypothesize that in patients with severe polyps (perhaps patients with asthma or Samter's Triad) more extensive first surgery may be beneficial.

In practical terms, with respect to the frontal sinus we are talking about the early or even initial use of Draf 3 surgery in selected patients. It would seem incongruous to recommend more 'radical' approaches to the frontal sinus while employing more standard procedures in the maxillary, sphenoid or ethmoid. Surgery incorporating the idea of "nasalization" has enjoyed popularity in some centers in Europe. One of many concerns associated with extensive surgical resection is the so-called 'empty nose syndrome'. These sort of extensive operations may not be equally useful as all climatic circumstances.

Implicit in the discussion of Draf 3 procedures at initial presentation in selected patients is the issue of who should be performing this surgery and caring for these patients. Draf 3 procedures may not be widely performed by general otolaryngologists, and are more likely to be well performed by specialist rhinologic surgeons. There is also a clear riskbenefit concern here. More extensive surgery may reduce the chances of polyp recurrence, but certainly not eliminate it. This "improvement" needs to be balanced against the risks of more extensive operative intervention. This inflammatory-load hypothesis remains at present simply that: an hypothesis. It does, however, highlight some interesting potential future trends, as well as reflecting a pendulum-like swing of surgical thinking away from the almost universal mantra of conservative surgery first in all patients.

\section{Conclusion}

Failed endoscopic sinus surgery continues to be a significant problem both in terms of its economic consequences 
but also with respect to significant patient quality of life issues. Good results required good equipment, appropriate surgical skills and the ability to devote sufficient time to sometimes challenging surgical cases. At a very basic level we also need to select patients for surgery who have a reasonable likelihood of post operative symptomatic improvement-a dilemma well illustrated by the difficulties of sinus surgery for patients with chronic facial pain and headache. In other patients, immune deficiency needs to be considered and excluded in patients who have failed surgery. A further small group of patients may also exist who might benefit from more extensive surgical intervention, perhaps earlier in the natural history of the disease.

Disclosure No potential conflict of interest relevant to this article was reported.

\section{References}

Papers of particular interest, published recently, have been highlighted as:

- Of importance

•- Of major importance

1. Graham SM, Nerad JA. Orbital complications in endoscopic sinus surgery using powered instrumentation. Laryngoscope. 2003;113(5):874-8.

2. Shen PH, Weitzel EK, Lai JT, Wormald PJ, Ho CS. Intravenous esmolol infusion improves surgical fields during sevoflurane anesthetised endoscopic sinus surgery: a double-blind, randomized placebo controlled trial. Am J Rhinol Allergy. 2011;25(6): e208-11.

3. Anand VK. Epidemiology and economic impact of rhinosinusitis. Ann Otol Rhinol Laryngol. 2004;193:3.

4. Owings MF, Kozak LJ. Ambulatory and inpatient procedures in The United States 1996. Vital Health Stat 13. 1998;139:1-119.

5. Bolger WE, Butzin CA, Parsons DS. Paranasal sinus bony anatomic variations and mucosal abnormalities: CT analysis for endoscopic sinus surgery. Laryngoscope. 1991;101:56-64.

6. Jones NS, Strobl A, Holland I. A study of CT findings in 100 patients with rhinosinusitis and 100 controls. Clin Otolaryngol. 1997;22:42-51.

7. Cook PR, Nishioka GJ, Davis WE, McKinsey JP. Functional endoscopic sinus surgery in patients with normal computed tomography scans. Otolaryngol Head Neck Surg. 1994;110:505-9.

8. - Halawi A, Mailey J, Graham SM. Craniofacial osteoma. Clinical presentation and patterns of growth. Am J Rhinol Allergy (In Press). Provides a contemporary appreciation of growth prospects of osteomas.

9. Stammberger $\mathrm{H}$, Wolf $\mathrm{G}$. Headaches and sinus disease: the endoscopic approach. Ann Otol Rhinol Laryngol. 1988;143:3-23.

10. West B, Jones NS. Endoscopy-negative, computed tomographynegative facial pain in a nasal clinic. Laryngoscope. 2001;111: 581-6.

11. Paulson EP, Graham SM. Neurologic diagnosis and treatment in patients with computed tomography and nasal endoscopy negative facial pain. Laryngoscope. 2004;114:1992-6.
12. Welge-Luessen A, Hauser R, Scmoid N, Kappos L, Probst R. Endonasal surgery for contact point headaches: a 10-year longitudinal study. Laryngoscope. 2003;113:2151-6.

13. Behin F, Behin B, Bigal ME, Lipton RE. Surgical treatment of patients with refractory migraine headaches and intra-nasal contact points. Cephalalgia. 2005;25:439-43.

14. • Guyuran B, Kreigler JS, Davis J, Amini SB. Five-year outcome of surgical treatment of migraine headaches. Plast Reconstr Surg. 2011;127:603-608. Highlights a potential role for surgery in a controversial condition.

15. Chee L, Graham SM, Carothers DG, Ballas ZK. Immune dysfunction in refractory sinusitis in a tertiary setting. Laryngoscope. 2001;III:233-5.

16. - Alqudah M, Graham SM, Ballas ZK. High prevalence of humoral immunodeficiency patients with refractory chronic rhinosinusitis. Am J Rhinol Allergy. 2010;24(6):409-412. Emphasizes the need to exclude immune dysfunction as a cause of recurrent sinus disease.

17. Graham SM. The maxillary sinus. Otorhinolaryngologist. 2012;5(2): 76-9.

18. •• Alby S, Gocea A, Necula S. Simultaneous inferior middle meatal antrostomies in the treatment of the severely diseased maxillary sinus. Am J Rhinol Allergy. 2011;25:e80-e85. Suggests efficacy of a comparatively simple surgical modification in improving post-operative outcomes.

19. Cho DY, Hwang PH. Results of endoscopic maxillary mega antrostomy in recalcitrant maxillary sinusitis. Am J Rhinol. 2008;22(6):658-62.

20. Sathanathar S, Naggonkar S, Paleris V, et al. Canine fossa puncture and clearance of the maxillary sinus for the severely diseased maxillary sinus. Laryngoscope. 2005;115:1026-9.

21. Robinson S, Wormald PJ. Patterns of innervation of the anterior maxilla: a cavader study with relevance to canine fossa puncture of the maxillary sinus. Laryngoscope. 2005;115:1785-8.

22. Singha D, Douglas R, Robinson S, et al. The incidence of complications using new landmarks and a modified technique of canine fossa puncture. Am J Rhinol. 2007;21:316-9.

23. Seiberling K, Ooi E, Miin Yip J, et al. Canine fossa trephine for the severely diseased maxillary sinus. Am J Rhinol Allergy. 2009;23(6):615-8.

24. $\bullet$ Lee JY et al. Is the canine fossa puncture approach really for the severely diseased maxillary sinus during endoscopic sinus surgery? Laryngoscope. 2011;118:1083-1186. Addresses a controversial issue in a well performed study.

25. Feldt BA, McMains KC, Weitzel EK. Cadaveric comparison of canine fossa versus trans-nasal maxillary sinus access. Int Forum Allergy Rhinol. 2011;1:183-186.

26. Wreesman VB, Fokkens WJ, Knegt PP. Refractory chronic sinusitis: evaluation of symptoms improvement after Denker's procedure. Otolaryngol Heak Neck Surg. 2001;125:495-500.

27. Viedlar WJM, Wressman VB, Van der Muelen FW, et al. Repetitive endoscopic sinus surgery failure. A role for radical surgery? Otolaryngol Head Neck Surg. 2006;134:586-91.

28. - Alqudah M, Graham SM. Modified osteoplastic approach for frontal sinus disease. Ann Otol Rhinol Laryngol. 2012;121(3): 192-196. Highlights a continuing role for external approach major surgery for a select group of patients.

29. Bassiouni A, Wormald PJ. Role of frontal sinus surgery in nasal polyp recurrence. Laryngoscope. 2012 [Epub ahead of print].

30. - Bassiouni A, Naidoo Y, Wormald PJ. When FEES Fails: The inflammatory Load hypothesis in refractory chronic rhinosinusitis. Laryngoscope 122:460-466. Introduces an interesting "hypothesis" for treatment of patients with extensive disease. 